\title{
The Bounds of Subjecthood: Evidence from Instruments*
}

\author{
SCOTT GRIMM \\ Stanford University
}

Canonical subjects of eventive predicates are agentive and animate-so goes one of the overarching, and idealized, generalizations of argument realization. As robust as this generalization is, caveats are always made (e.g. Comrie 1989:107,128), in part due to a set of recalcitrant counter-examples, known as "instrumental subjects", which violate this generalization on both counts. A paradigm case of the phenomenon is shown in (1): the entity which appears as an instrument in (1a) also appears as a subject in (1b), although it is neither agentive nor animate.

(1) a. Marvin hit the horse with a stick.

b. The stick hit the horse.

(from Schlesinger 1989)

Inasmuch as theories of argument realization base themselves on the generalization tout court that agents are subjects, "instrumental subjects" straightaway pose difficulties. I will demonstrate that a more articulated and systematic approach to argument structure dissolves the difficulties posed by "instrumental subjects" and their kin. An analysis is developed in which the restrictions on the realization of instrumental subjects are a natural consequence of a novel approach to modeling argument structure, loosely based on the proto-role theory of Dowty 1991. In this framework, such subjects ultimately need not be viewed as anomalies, but simply as possible subjects, albeit often the limiting case.

\section{Delimiting Inanimate Actors}

A consensus has emerged that while instruments are perhaps a class of arguments,

* This paper has benefitted from the comments of Patricia Amaral, Marie-Catherine de Marneffe and Nola Stephens. I would also like to thank Beth Levin and Henk Zeevat for current and former advising, respectively. 


\section{Scott Grimm}

the classification of certain subjects as "instrumental subjects" is amiss; DeLancey 1991, for one, claims the notion of "instrumental subject" is a "spurious class", essentially an unwanted artifact of theoretical assumptions from the early days of Case Grammar. For the present, it suffices to notice that there is not an equivalence between NPs which appear as instruments, i.e. in a 'with' phrase, and those which can be realized as subjects. First, the putative class of instrumental subjects is not determined by the entities able to occupy the canonical instrument position-some instruments simply cannot be realized as subjects, as shown in (2).

(2) a. Carl ate spaghetti with a fork.

b. *The fork ate spaghetti. (from Levin and Rappaport Hovav 1988)

In the other direction, the instrumental subject class cannot be characterized by the set of non-agent entities realized as subjects of eventive predicates, for these need not be instruments. For instance, natural forces, as in (3), neither share the syntactic behavior of instruments, i.e. do not appear in a 'with' phrase, nor are they conceptually similar to canonical instruments.

(3) The wind opened the door.

(from Fillmore 1968)

Therefore, the class of permissible inanimate subjects does not correspond to the class of instruments, and a more general solution is needed.

In coping with these phenomena, the last decades have seen a number of distinctions arise, all of which are in some sense correct, but none of which alone has proven sufficient. First, as (4) demonstrates, what can be realized as a subject of a verb depends in part upon the nature of the entity.

(4) a. The worker moved the dirt with the crane/shovel.

b. The crane/*shovel moved the dirt. (modified from Levin 1993)

A distinction has been made between "intermediary" and "facilitating" instruments (see Levin and Rappaport Hovav 1988 and references therein as well as the "primary"/"secondary" tool distinction of Nilsen 1973). Intermediary instruments designate entities able "to perform the action independently", whereas facilitating instruments designate those without such abilities. Under this account, certain verbs would permit intermediary instruments as subjects, e.g. (4), while others select only for facilitating instruments, e.g. (2), thus the acceptability patterns of (4) and (2) follow.

Yet, even more factors play a role, as in (5), where the same entity qualifies as a subject of one predicate, but not of another.

(5) The bullet killed/*murdered the president.

(from Schlesinger 1989) 
The acceptability pattern of (5) does not hinge on whether an instrument can perform the action independently, rather on expectations associated with the predicate, namely that 'murder' involves some degree of intentionality. Accordingly, while a bullet can be held responsible for a death, it is less easily conceived that a bullet can be held responsible for a murder.

A third point of discussion has been the overlap between causes and instruments. Some accounts have claimed that sentences such as those below possess "instrumental" subjects.

(6) The bomb suddenly exploded.

(Nilsen 1973)

(7) The rust has eaten away at the lock.

(Schlesinger 1989)

(8) The wind opened the door.

(Fillmore 1968)

While DeLancey 1991 is correct in asserting that such examples are simply not instruments, there is an affinity between these inanimate subjects and the standard “instrumental subject", e.g. (1b). More generally, Comrie 1989:59 has noted that agents, forces, instruments, as well as patients partake of a continuum of control, "the labels representing different points along this continuum." Accordingly, any proposed solution of instrumentals should demonstrate how they are related to the other notions of forces and agents as well as patients.

The above data indicate the desiderata a solution to this problem should satisfy. First and foremost, the patterns of distribution should be accounted for: we should be able to predict which inanimate entities can become subjects of which eventive predicates. Second, the solution should be related to larger issues of argument realization, such as how inanimate actors are related to more canonical agents and under what conditions inanimate actors can take priority as subjects. I now provide an analysis which attempts to meet these two criteria explicitly.

\section{The Lattice of Agency}

The above discussion motivated the need for a framework capable of modeling the interaction of the verbal and nominal domain with respect to argument realization. Such a system has been previously developed in Grimm 2005 where it was applied to account for case-marking patterns. Case systems are found to be sensitive to both agency, e.g. in ergative systems (Mohanan 1994), and animacy, e.g. in differential object marking systems (see Aissen 2003 and references therein). Thus, while the system presented below is independently motivated for treating case systems, as a corollary it yields a resolution to the problem of the instrumental subject.

\subsection{Agency and the Verbal Domain}




\section{Scott Grimm}

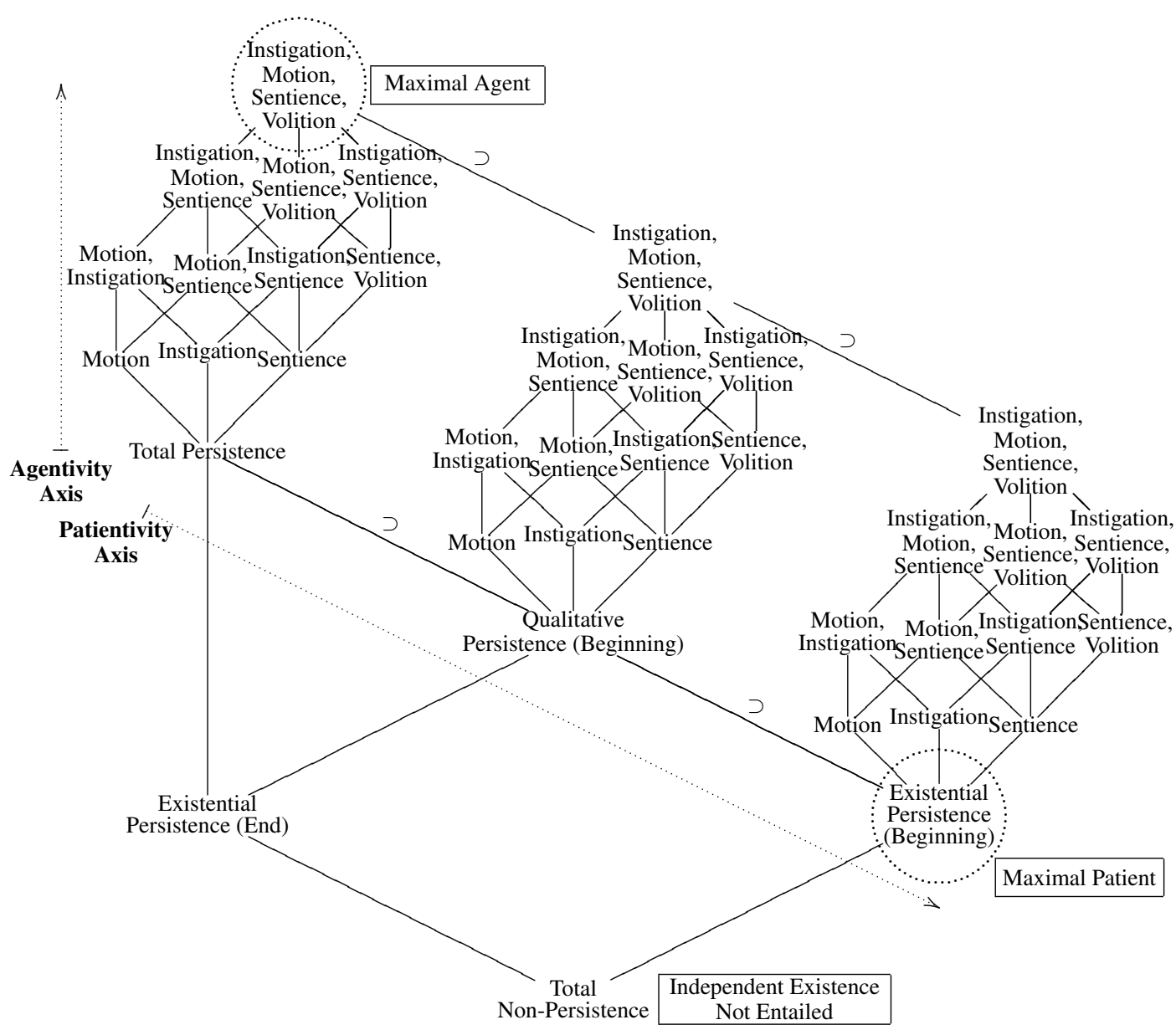

Figure 1: The Agency Lattice

The model of argument structure developed here assumes a set of event-based properties entailed by the verb referring to modes of participation in events: instigation, motion, sentience, volition, and different degrees of persistence. A predicate entails instigation only when the event described is necessarily brought about by one of its arguments. Motion is entailed just in case an argument is required to be in motion. Sentience designates conscious involvement in the event while volition designates deliberate engagement in the event. Agents, then, will typically possess one or more of these properties.

Persistence tracks how entities change throughout the event. An entity can persist in two ways: existentially, that is, it exists perpetually throughout the event/state, or qualitatively-i.e., it persists in all its particulars. Either of these can obtain at the 


\section{The Bounds of Subjecthood: Evidence from Instruments}

beginning and/or the end of the event, giving the following set of properties: existential persistence (beginning), existential persistence (end), qualitative persistence (beginning), and qualitative persistence (end).

The above properties are represented as features in privative opposition. Logical entailments among the eight features constrain the combinations possible. For instance, volition entails sentience, since only sentient beings are capable of volition, and -existential persistence (end) entails - qualitative persistence (end), since if an entity does not exist at the end of the event, clearly none of its qualities do either. Consequently, if an entity possesses qualitative persistence (end), it persists entirely throughout the event and thus possesses all four persistence features, referred to as total persistence for convenience. The remaining combinations can then be given greater structure. Ordering the properties and their combinations by inclusion, modulo the mentioned impossibilities, yields a partial order, which can be further structured as a lattice, shown in figure 1, referred to henceforth as the agency lattice.

This lattice exhibits the possible space of argument types with respect to agency. The particular argument structure of a given predicate can be mapped upon this space. The opposition between agents and patients falls out from this feature system in that agents will possess total persistence along with a number of other agency properties while patients will generally possess no properties save existential persistence (beginning) and possibly qualitative persistence (beginning). The agency lattice delivers several additional properties. First, the privative opposition is conspicuous in the structure: the highest node contains all the features, corresponding to a full agent possessing all the properties, while the lowest contains none, not entailing even independent existence, corresponding to, e.g. arguments of negative existence statements or incorporated/cognate objects ("sing a song"). Second, agents are upwards closed in the lattice while patients are downward closed. That is to say, if some node $x$ of the agency lattice is considered an agent (patient) relative to a given predicate, then all the connected nodes higher (lower) than $x$ are as well. Thus what counts as an agent or a patient is characterized by a region, which directly addresses the fact that the agent position of a given predicate can be instantiated by a variety of types of "agentive" participants, as was seen in the examples of the last section. Finally, subject selection is accomplished merely by selecting the argument which has more entailments, i.e. if argument $\mathrm{A}$ lives on a node which dominates argument $\mathrm{B}$, then argument $\mathrm{A}$ is selected as subject. In sum, given a specified set of entailments for each argument of a predicate, this structure outputs the connected region consistent with those arguments and the structural relation that 


\section{Scott Grimm}

holds between the arguments of the predicates (if more than one) corresponding to their relative semantic prominence, in turn determining subject and object selection.

\subsection{Animacy and the Nominal Domain}

A compatible structure for the nominal property of animacy can be constructed. Along with the standard distinction between humans, animals, and inanimates, it is helpful to distinguish between immobile entities (e.g. locations) and mobile objects, i.e. those capable of moving or being moved. The features human, sentient and mobile differentiate between humans and non-humans, animates and inanimates, and inanimates which can move or be moved and those which cannot, respectively. Further, (4) showed the necessity of distinguishing inanimates capable of (semi-)autonomous activity. Such a distinction has been observed independently of the instrumental subject literature and I will be adopting the broader notion of potent from Chafe 1970, defined for an entity which "has, or is conceived to have, its own internal power" (Chafe 1970:109).

The same procedure that was used in the verbal domain to provide structure can be applied here: these distinctions are represented by features which are then given greater structure. Various entailments constrain the possible combinations: human entails sentient which in turn entails both potent and mobile. These properties and their possible combinations form a partial order, which corresponds to a version of the familiar animacy scale:

(9) $\{\emptyset\} \subset\{$ mobile $\},\{$ potent $\} \subset\{$ mobile, potent $\} \subset\{$ mobile, potent, sentient $\}$ $\subset\{$ mobile, potent, sentient, human $\}$

(10) inanimate $<$ mobile inanimate, potent inanimate $<$ mobile and potent inanimate (e.g. natural force, autonomous machine) $<$ animate $<$ human

This animacy scale can then be combined with the agency lattice (formally, taking their cartesian product; informally, inserting the agency lattice on each node of the animacy structure). The unified agency and animacy structure, of which the total persistence branch is shown in figure 2, establishes the possible instantiations of argument structure property combinations by nominal entities. The conjunction of the two structures is constrained by removing incompatible feature sets. For example, instigation by definition requires an argument which is capable of bringing about the event designated by the predicate. If an entity is powerless to bring about the event, then it can not satisfy the instigation entailment. Therefore, any entity which is to satisfy an instigation entailment must qualify as potent in a sense relevant to the predicate. As a consequence, no node of the lattice will contain instigation without potent. By similar reasoning, the lattice will not contain motion without mobile, 
The Bounds of Subjecthood: Evidence from Instruments

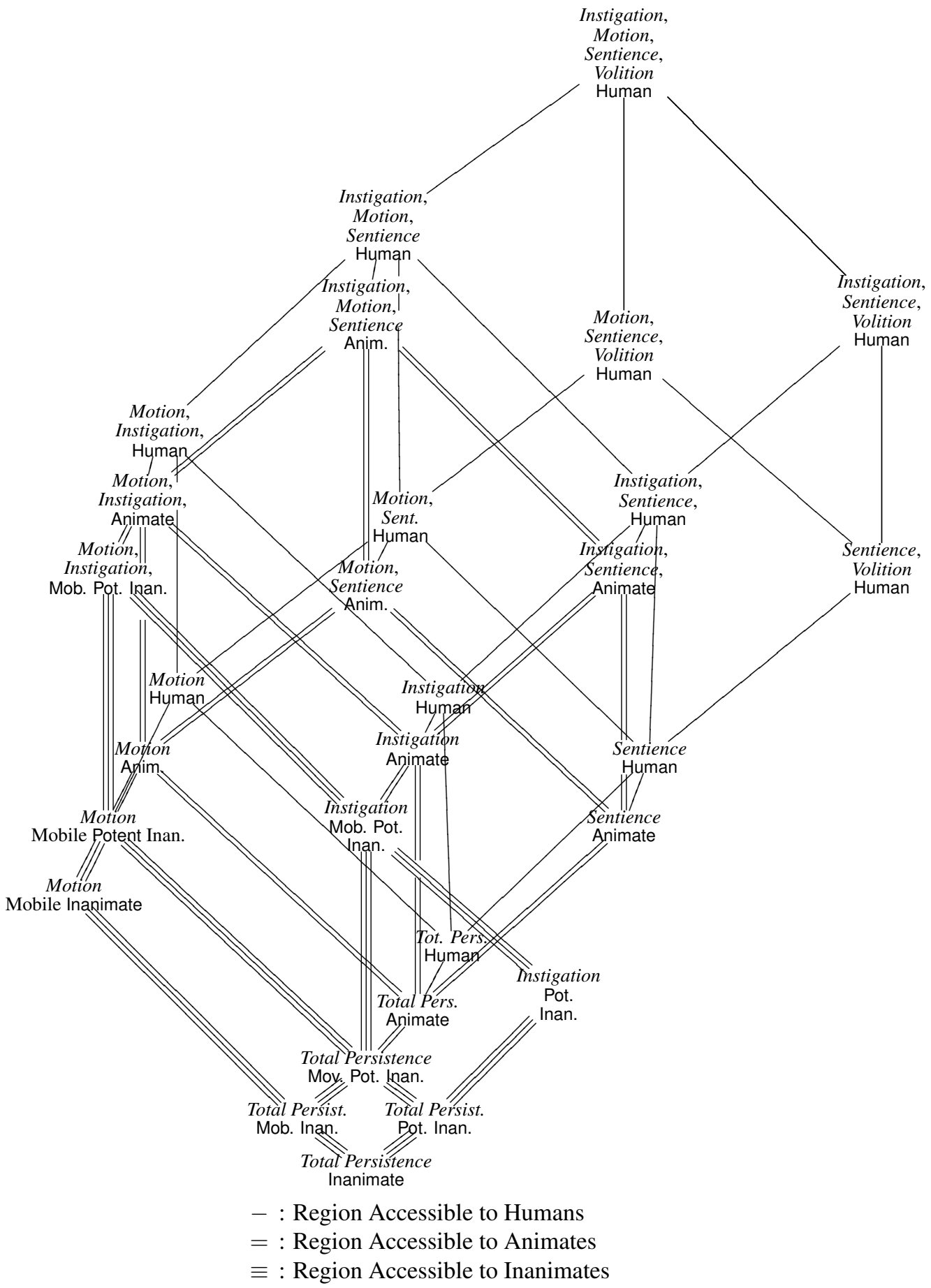

Figure 2: Product of Agency Lattice and Animacy Hierarchy 


\section{Scott Grimm}

sentience without sentient, nor volition without human. In general, the higher in the lattice, the fewer entities qualify to instantiate.

This structure then produces the acceptability patterns of the instrumental subject data. Broadly, a given verb puts a set of entailments into play for its arguments; if the given entity possesses the features needed to satisfy the entailments on the argument, then the entity can serve as a subject, otherwise not. In terms of the lattice, the argument structure of a predicate restricts the region of the lattice its subject may inhabit by the properties that the predicate entails. This in turn restricts the types of nominals satisfying the predicate's requirements to just those which are consistent with the region of the lattice corresponding to the predicate's subject. Canonical instruments, as well as other inanimate actors, are limited to one connected region of the lattice. When a predicate's entailments coincide with this region, then such instruments may be realized as subjects.

\section{An Account of Inanimate Actors}

The framework developed above can now be put to work on the problems outlined in section 1. First, it can be demonstrated under what circumstances inanimate actors appear, as in the first example discussed, repeated here as (11).

(11) a. Marvin hit the horse with a stick.

b. The stick hit the horse.

The nominal 'stick' possesses the feature mobile, thus it is compatible with the region of the structure indicated by the dotted line in figure 3 , the relevant sublattice of figure 2. In examining the entailments of the predicate, it is sufficient to note that the subjects of 'hit' typically are in motion and are unaffected by the event, thus entailing the feature motion and total persistence. Since the focus is on the impact, instigation is not at issue ${ }^{2}$. Thus, as figure 3 graphically describes, the region established by the entity's properties coincides with the predicate's entailments, and therefore the combination is acceptable.

The next example, repeated here as (12), holds the predicate constant while the nominal varies, displaying the manner in which the properties of the nominal entity determine its acceptability as a subject.

\footnotetext{
${ }^{2}$ While most occurrences of "hit" implicate a degree of instigation, this is not strictly entailed, as examples of the following sort illustrate:

(1) "The tank accidentally backed into a tree and a branch hit the mounted, unmanned machine gun, causing it to fire, Curtin said."

Www.signonsandiego.com/uniontrib/20050911/news 1 n11 armydead.html
}

In these cases, tests of prior activity such as "what the branch did was hit the gun" fail. 


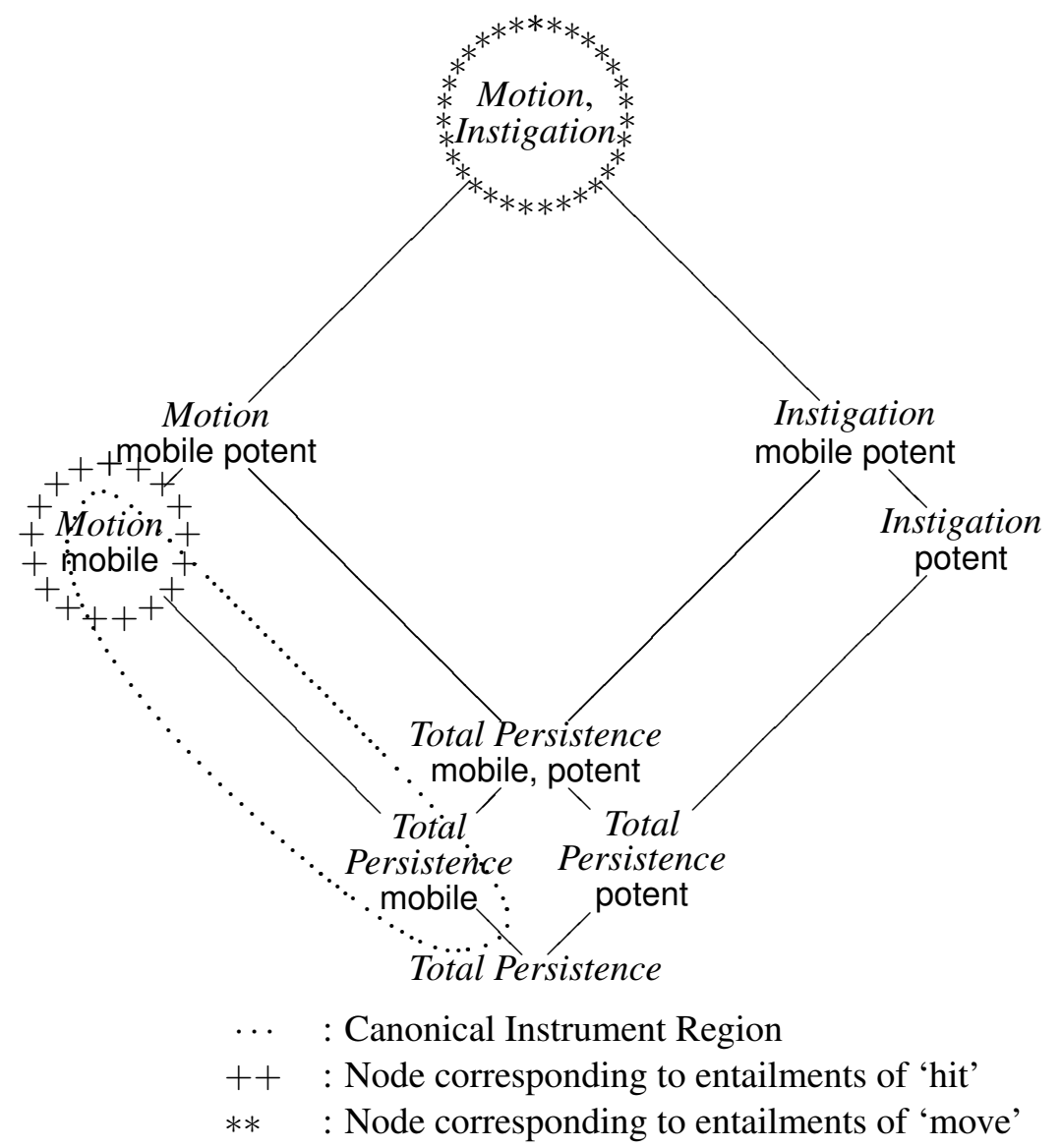

Figure 3: Sublattice of Unified Agency and Animacy Lattice

(12) a. The worker moved the dirt with the crane/shovel.

b. The crane/*shovel moved the dirt.

The properties of 'shovel' are identical to those of 'stick', only possessing the feature mobile. 'Crane', however, as a semi-autonomous machine qualifies for both mobile and potent. The verbal entailments for 'move' clearly include motion and total persistence. Further, the transitive use of 'move', move $X$ from $Y$ to $Z$, requires some sustained activity bringing about the determined relocation, thereby entailing instigation. To satisfy the instigation entailment, an entity must possess the feature potent, which 'crane' does and 'shovel' fails to do, as seen in figure 3.

A third example, repeated as (13), holds the entity constant, demonstrating how minimally different predicates determine the acceptability of inanimate subjects. 


\section{Scott Grimm}

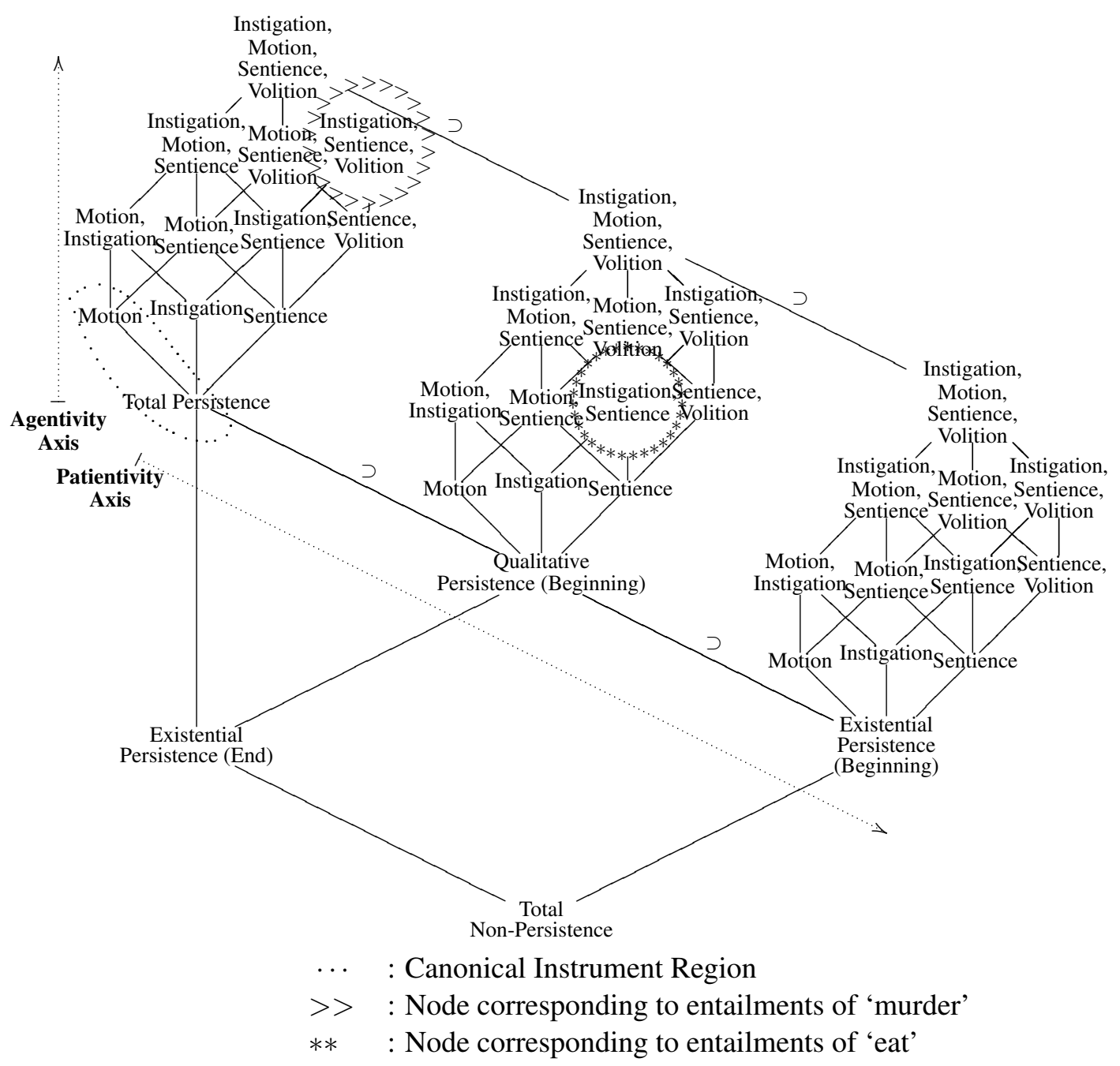

Figure 4: Entailments for 'murder' and 'eat'

(13) The bullet killed/*murdered the president.

As a projectile, 'bullet' is clearly mobile, but also, if construed as in motion, it qualifies as possessing a type of 'internal power', viz. the force associated with its kinetic energy, thereby qualifying for potent (see Kearns 2000:241). However, 'murder' has more stringent requirements than 'kill', for 'murder', as has long been observed, entails volitionality. While 'bullet' clearly fulfills the requirements of 'kill', since it cannot satisfy the sentience entailment, and in turn the volitionality 
entailment, it fails to fulfill the requirements for 'murder', shown in figure 4.

A similar situation holds in (14), repeated from above.

(14) a. Carl ate spaghetti with a fork.

b. *The fork ate spaghetti.

Previous analyses claimed the unacceptability of (14b) stemmed from the fact that verbs such as 'eat', as well as 'see', only select for facilitating instruments, such as 'fork'. Yet, this explanation leaves open the question of why this particular set of verbs does not select for instrumental subjects. In terms of the lattice, ingestive verbs clearly entail sentience and are also among the set of verbs requiring an affected agent (see Saksena 1980), along with perceptual and psychological verbs. Such verbs locate their subject argument on the qualitative persistence (beginning) branch of the lattice (i.e. qualitatively affected). Since the properties and lattice location of 'fork' are equivalent to those of 'shovel' or 'stick', it clearly cannot satisfy the entailments of 'eat', as figure 4 illustrates.

The analysis developed here provides an explanation which grounds these verbs' inability to take instruments as subjects in their overall semantics. To qualify as a subject the argument must be sentient and affected in the relevant manner. No canonical, or readily conceivable, instrument is able to be affected in place of the affected agent. Consequently, instruments do not qualify as subjects of these verbs.

Having established an analysis for what have been the traditional core cases of instrumental subjects, this same machinery can account for causes and natural forces, such as those repeated in (15)-(17).

(15) The bomb suddenly exploded.

(16) The rust has eaten away at the lock.

(17) The wind opened the door.

Causes, such as in (15) and (16), possess the feature potent. Natural forces are capable of both autonomous activity and motion, possess the features potent and mobile, and may serve as subjects of predicates such as 'open', 'move', etc. Thus, causes, instruments and natural forces are all contiguous, demonstrating that indeed such notions can be thought of as a continuum.

\section{Conclusion}

The problem of instrumental subjects has endured in part because of a need for a sufficiently fine-grained system. Recasting the approach of Dowty 1991 into one set of features and hierarchized in a lattice has led to a structured framework able 


\section{Scott Grimm}

to interact with the animacy hierarchy, accounting for the heterogeneous set of restrictions on subject selection and predicting the distribution of inanimate subjects.

\section{References}

Aissen, Judith. 2003. Differential Object Marking: Iconicity vs. Economy. Natural Language and Linguistic Theory 21:435-483.

Chafe, Wallace. 1970. Meaning and the Structure of Language. Chicago: University of Chicago Press.

Comrie, Bernard. 1989. Language Universals and Linguistic Typology. Second Edition. Chicago: University of Chicago Press.

DeLancey, Scott. 1991. Event Construal and Case Role Assignment. In L. A. Sutton, C. Johnson, and R. Shields, eds., Proceedings of Berkeley Linguistics Society 19:338-53.

Dowty, David. 1991. Thematic Proto-Roles and Argument Selection. Language 67:547-619.

Fillmore, Charles. 1968. The Case for Case. In E. Bach and R. Harms, eds., Universals in Linguistic Theory, 1-88. New York: Holt, Rinehart and Winston.

Grimm, Scott. 2005. The Lattice of Case and Agentivity. Master's Thesis. Institute for Logic, Language and Computation: Universiteit van Amsterdam.

Kearns, Kate. 2000. Semantics. London: Macmillan Press.

Levin, Beth. 1993. English Verb Classes and Alternations: A preliminary investigation. Chicago: University of Chicago Press.

Levin, Beth and Malka Rappaport Hovav. 1988. Nonevent -er Nominals: A Probe into Argument Structure. Linguistics 26:1067-1083.

Mohanan, Tara. 1994. Argument Structure in Hindi. Stanford, CA: CSLI.

Nilsen, Don. 1973. The Instrumental Case in English. The Hague: Mouton.

Saksena, Anuradha. 1980. The Affected Agent. Language 56:812-826.

Schlesinger, I. M. 1989. Instruments as Agents: On the Nature of Semantic Relations. Journal of Linguistics 25:189-210.

Scott Grimm

Stanford University

Department of Linguistics

Stanford CA 94305-2150

sgrimm@stanford.edu 